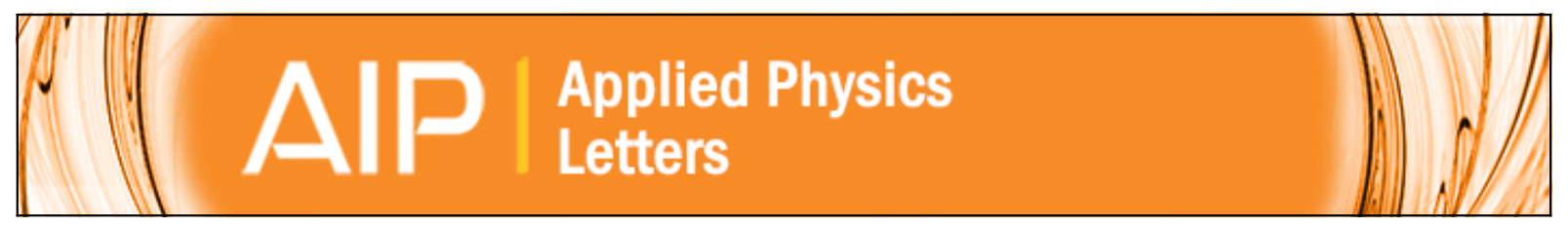

\title{
Impurity-free disordering mechanisms in GaAs-based structures using doped spin-on silica layers
}

Prakash N. K. Deenapanray, Bin Gong, R. N. Lamb, A. Martin, L. Fu, H. H. Tan, and C. Jagadish

Citation: Applied Physics Letters 80, 4351 (2002); doi: 10.1063/1.1484244

View online: http://dx.doi.org/10.1063/1.1484244

View Table of Contents: http://scitation.aip.org/content/aip/journal/apl/80/23?ver=pdfcov

Published by the AIP Publishing

\section{Articles you may be interested in}

Towards a better understanding of the operative mechanisms underlying impurity-free disordering of GaAs:

Effect of stress

J. Vac. Sci. Technol. B 21, 198 (2003); 10.1116/1.1535927

Atomic relocation processes in impurity-free disordered $p$-GaAs epilayers studied by deep level transient spectroscopy

Appl. Phys. Lett. 81, 3573 (2002); 10.1063/1.1519728

Influence of dielectric deposition parameters on the In $0.2 \mathrm{Ga} 0.8 \mathrm{As} / \mathrm{GaAs}$ quantum well intermixing by impurity-free vacancy disordering

J. Appl. Phys. 92, 1386 (2002); 10.1063/1.1486027

Dependence of band gap energy shift of In $0.2 \mathrm{Ga} 0.8$ As/GaAs multiple quantum well structures by impurity-free vacancy disordering on stoichiometry of $\mathrm{SiO} x$ and $\mathrm{SiN} x$ capping layers

J. Appl. Phys. 91, 4256 (2002); 10.1063/1.1448878

Novel impurity-free interdiffusion in GaAs/AIGaAs quantum wells by anodization and rapid thermal annealing Appl. Phys. Lett. 70, 1269 (1997); 10.1063/1.118549

\section{AIP $\mid$ APL Photonics \\ APL Photonics is pleased to announce Benjamin Eggleton as its Editor-in-Chief}

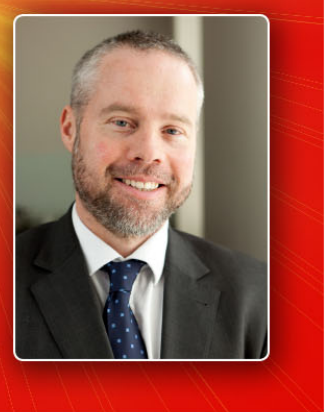




\title{
Impurity-free disordering mechanisms in GaAs-based structures using doped spin-on silica layers
}

\author{
Prakash N. K. Deenapanray ${ }^{\mathrm{a})}$ \\ Department of Electronic Materials Engineering, Research School of Physical Sciences and Engineering, \\ The Australian National University, Canberra, ACT 0200, Australia \\ Bin Gong and R. N. Lamb \\ Surface Science Centre and Technology, School of Chemistry, University of New South Wales, Sydney, \\ NSW 2052, Australia \\ A. Martin \\ Laboratoire de Physique des Interfaces et Couches Minces, Ecole Polytechnique, \\ 91128 Palaiseau Cedex, France \\ L. Fu, H. H. Tan, and C. Jagadish \\ Department of Electronic Materials Engineering, Research School of Physical Sciences and Engineering, \\ The Australian National University, Canberra, ACT 0200, Australia
}

(Received 11 January 2002; accepted for publication 11 April 2002)

\begin{abstract}
We have used photoluminescence, deep level transient spectroscopy and x-ray photoelectron spectroscopy to investigate the mechanisms of impurity-free disordering in GaAs-based structures using doped spin-on silica layers. We demonstrate that $V_{\mathrm{Ga}}$ is efficiently converted into arsenic-antisite, $\mathrm{As}_{\mathrm{Ga}}$, related defects (EL2-type defects) when the GaAs layer is under compressive stress. We propose that the efficient formation of EL2-type defects reduces the efficiency of impurity-free interdiffusion of GaAs/AlGaAs quantum wells. (c) 2002 American Institute of Physics. [DOI: 10.1063/1.1484244]
\end{abstract}

Impurity-free disordering (IFD) using dielectric capping layers has been used to modify the optical properties of GaAs-based materials for monolithic integration of devices with different functionalities. ${ }^{1}$ This process introduces defects related to gallium vacancies, $V_{\mathrm{Ga}}$, arsenic interstitials, $\mathrm{As}_{i}$, and arsenic antisites, $\mathrm{As}_{\mathrm{Ga}}$ (i.e., EL2-type defects) in GaAs. ${ }^{2,3}$ We have also shown recently that the introduction of these defects is influenced by the stress imposed by the dielectric layer on the GaAs layer at the annealing temperature. ${ }^{4}$ In GaAs-based structures, IFD is a two-step mechanism, including the creation of $V_{\mathrm{Ga}}$ by outdiffusion of $\mathrm{Ga}$ atoms into the capping layer, and the diffusion of $V_{\mathrm{Ga}}$ away from the capping layer-GaAs interface. ${ }^{5}$ Experimental evidence shows that the second step is stress dependent. ${ }^{5-7}$ However, the relative importance of the two steps is not known.

Doped plasma-enhanced chemical vapor deposited $\mathrm{SiO}_{2}$ (Ref. 8) and spin-on glass (SOG) (Refs. 9-11) layers have been used to control the extent of interdiffusion of GaAsbased structures. These silica layers are inexpensive and readily available commercially, and their properties, including porosity and doping, can be controlled. The relative importance of the two steps that drive disordering in GaAsbased structures can be addressed by using doped and undoped SOG. In this letter, we demonstrate that $V_{\mathrm{Ga}}$ vacancies created by the outdiffusion of $\mathrm{Ga}$ atoms into the silica capping layer may not contribute to the interdiffusion process if they are efficiently converted into EL2-type defects.

Epitaxial GaAs layers of (100) orientation doped with

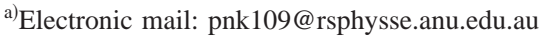

$1 \times 10^{16} \mathrm{Si} / \mathrm{cm}^{3}$ were grown by metalorganic chemical vapor deposition (MOCVD). The epitaxial layers were spin coated with undoped, P-doped, and Ga-doped silicate SOG at 3000 rpm for $30 \mathrm{~s}$. The samples were baked at $400{ }^{\circ} \mathrm{C}$ for $15 \mathrm{~min}$ before rapid thermal annealing (RTA) at $900^{\circ} \mathrm{C}$ for $30 \mathrm{~s}$ under Ar flow. Details of Schottky barrier fabrication and deep level transient spectroscopy (DLTS) measurements have been described previously. ${ }^{2-4} \mathrm{X}$-ray photoelectron spectroscopy (XPS) was used to study the outdiffusion of Ga into the SOG layers. We used a VG ESCALAB 220- $i$ XL spectrometer using monochromatized $\mathrm{Al} K \alpha$ x rays and an Ar-ion gun for in-depth profiling. The in-depth profiles of $\mathrm{O}, \mathrm{Si}, \mathrm{Ga}, \mathrm{As}$, and $\mathrm{P}$ were measured by recording the $\mathrm{O} 1 s$, Si $2 p$, Ga $2 p_{3 / 2}$, As $3 d$, and $\mathrm{P} 2 p$ photoelectron spectra, respectively. SOG-induced impurity-free interdiffusion of a GaAs/ AlGaAs structure that contains two quantum wells (QWs) was investigated by low-temperature photoluminescence (PL). The nominal thickness of the QWs was $2.3 \mathrm{~nm}(\mathrm{QW} 1$, closer to surface) and $4.0 \mathrm{~nm}$ (QW2), and they were separated by $50 \mathrm{~nm} \mathrm{Al}{ }_{0.54} \mathrm{Ga}_{0.46}$ As barriers.

Figure 1 illustrates the PL spectra obtained from uncapped (dotted line) and oxide capped (solid line) samples with undoped, P-doped, and Ga-doped SOG layers following RTA at $900{ }^{\circ} \mathrm{C}$ for $60 \mathrm{~s}$. Two clearly resolved excitonic peaks that correspond to $n=1$ electron to heavy-hole transitions in the two QWs are observed in each case. The emission wavelength of the capped and annealed QWs is blueshifted, with the effect being most pronounced for P-doped SOG and least for Ga-doped SOG. The blueshift in excitonic emission from QWs is the result of the increase in band gap of GaAs QWs following $\mathrm{Al}-\mathrm{Ga}$ interdiffusion across the barrier-well interface. ${ }^{1,5-11}$ It is worth noting here that the larger blueshift for 


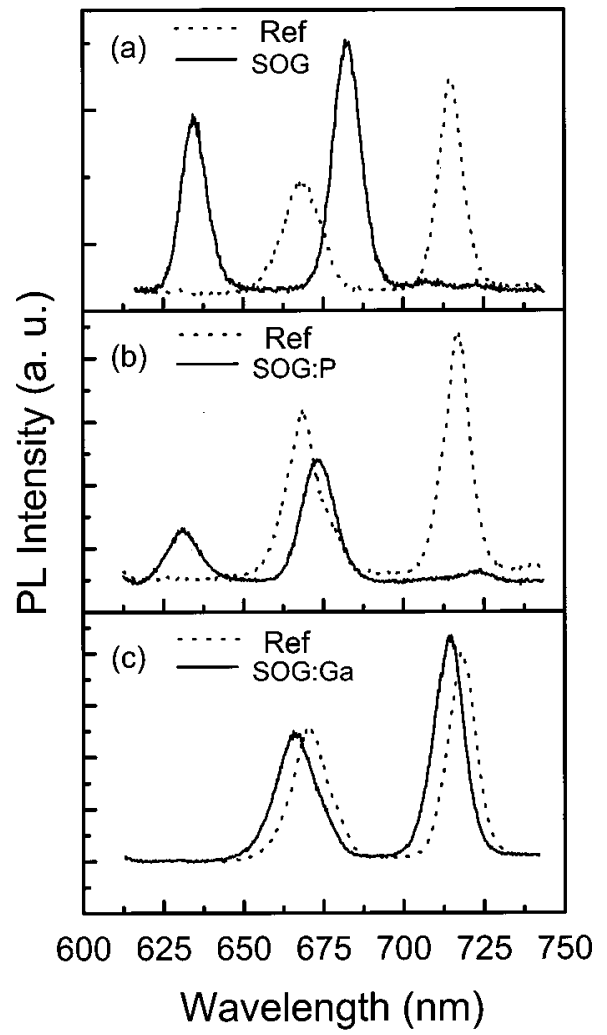

FIG. 1. PL spectra (solid curves) of the samples capped with (a) undoped, (b) P-doped, and (c) Ga-doped spin-on silica layers baked at $400{ }^{\circ} \mathrm{C}$ for 15 min and annealed at $900{ }^{\circ} \mathrm{C}$ for $60 \mathrm{~s}$. Spectra shown by dotted curves are from uncapped annealed samples (references).

QW2 from P-doped SOG compared to undoped SOG is consistent with the results of Rao et al., ${ }^{12}$ but is in contrast to those of Cusumano et al. ${ }^{8}$

Figure 2 shows the XPS in-depth profiles of $\mathrm{Ga}, \mathrm{O}, \mathrm{Si}$, As, and/or P in undoped, P-doped, and Ga-doped SOG layers. Figure 2(a) reveals the outdiffusion of Ga atoms into the undoped SOG, with most segregation taking place on the oxide side of the SOG/GaAs interface. This result is in qualitative agreement with the PL results in Fig. 1(a). The GaAs layer is under compressive stress during RTA, which makes the outdiffusion of $\mathrm{Ga}$ atoms into the SOG layer thermodynamically favorable. ${ }^{5}$ A comparison of Figs. 2(a) and 2(c) may explain the suppression of interdiffusion by the $\mathrm{Ga}-$ doped SOG layer. The Ga-doped SOG contains 6\%-7\% Ga after RTA. We have not observed any marked differences between the distributions of Ga within the Ga-doped SOG before and after RTA. Since this concentration exceeds the concentration of outdiffused Ga into the undoped SOG [Fig. 2(a)], there is a diffusion barrier against the segregation of Ga atoms into the Ga-doped SOG layer. Consequently, the interdiffusion process is suppressed as shown in Fig. 1(c). However, the slight blueshifts in PL emission of IFD QWs suggests that chemical reactions between GaAs and Gadoped SOG in a region confined to the SOG/GaAs interface may still produce some $V_{\mathrm{Ga}}$.

Figure 2(b) shows that the P-doped SOG layer contains $\sim 0.4 \%$ phosphorus after annealing. The doping concentration in our P-doped SOG is very similar to that used by Rao et al. ${ }^{12}$ but is about one order magnitude smaller than the $\mathrm{P}$ doping used by Cusumano et al. ${ }^{3}$ We can, therefore, con-

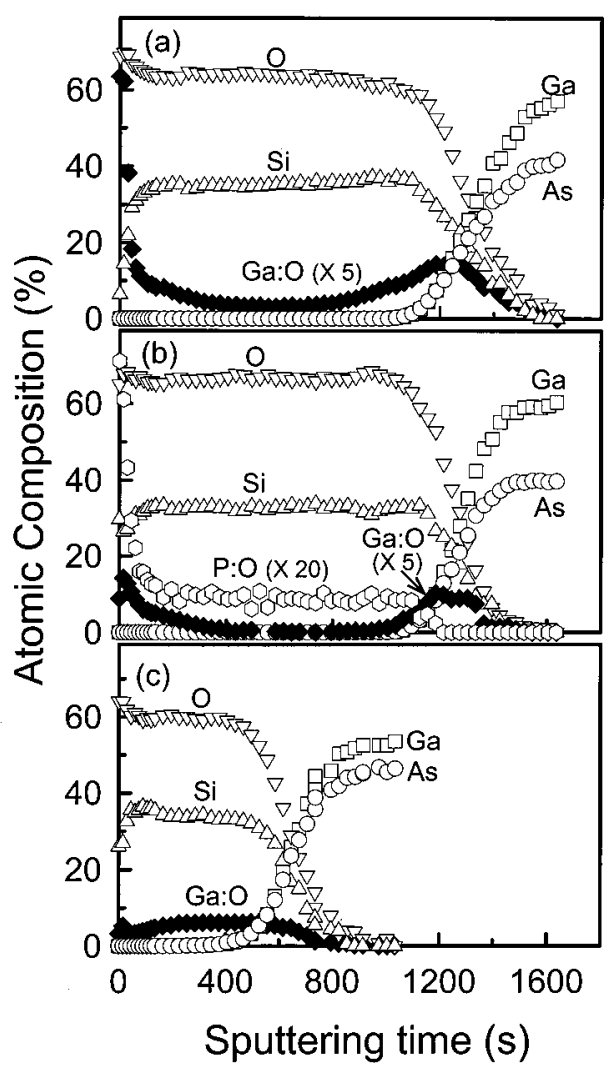

FIG. 2. XPS in-depth profiles of $\mathrm{Ga}, \mathrm{Si}, \mathrm{O}, \mathrm{As}$, or $\mathrm{P}$ in (a) undoped, (b) P-doped, and (c) Ga-doped spin-on silica layers annealed at $900{ }^{\circ} \mathrm{C}$. The outdiffusion of $\mathrm{Ga}$ into the silica capping layers was measured by monitoring the $\mathrm{Ga} 3 p_{3 / 2}$ photoelectron spectrum. The $\mathrm{Ga}$ in the oxide layers is bonded to oxygen.

clude that the concentration of phosphorus in P-doped SOG layers is a critical parameter to consider when designing IFD studies. Both the porosity of the SOG layer and the compressive stress that it imposes on the GaAs structure are expected to decrease with an increase of $\mathrm{P}$ doping. ${ }^{8}$ Rao et al. ${ }^{12}$ have attributed the larger interdiffusion in their QW structures by P-doped SOG compared to an undoped layer to increased solubility of $\mathrm{Ga}$ atoms in the former layer, but did not provide any experimental evidence to support their argument. A comparison of the results shown in Figs. 2(a) and 2(b) clearly demonstrates that this is not the case. Although there is outdiffusion of $\mathrm{Ga}$ atoms into the P-doped layer [Fig. 2(b)], the segregation effect is less than in undoped SOG [Fig. 2(a)]. Based on these results alone one would expect a larger concentration of $V_{\mathrm{Ga}}$, and hence larger interdiffusion, to be produced with undoped SOG. The results shown in Figs. 1 and 2 unambiguously show that although interdiffusion is related to the outdiffusion of $\mathrm{Ga}$ atoms into the capping layer, other processes, which have not been identified thus far, determine the extent of disordering.

In order to explain our results, we have studied the defects created in rapid thermally annealed $n$-GaAs epitaxial layers capped with the undoped and doped SOG layers. Figure 3 illustrates DLTS spectra after RTA for $n$-GaAs capped with undoped (dashed line), P-doped (solid line), and Gadoped (dotted line) SOG layers. IFD created four electron traps $S 1 \quad\left(E_{C}-0.23 \mathrm{eV}\right), \quad S 2 \quad\left(E_{C}-0.46 \mathrm{eV}\right), \quad S 3 \quad\left(E_{C}\right.$ $-0.72 \mathrm{eV})$, and $S 4\left(E_{C}-0.74 \mathrm{eV}\right)$. The concentration of defects created by Ga-doped SOG is similar to that obtained 


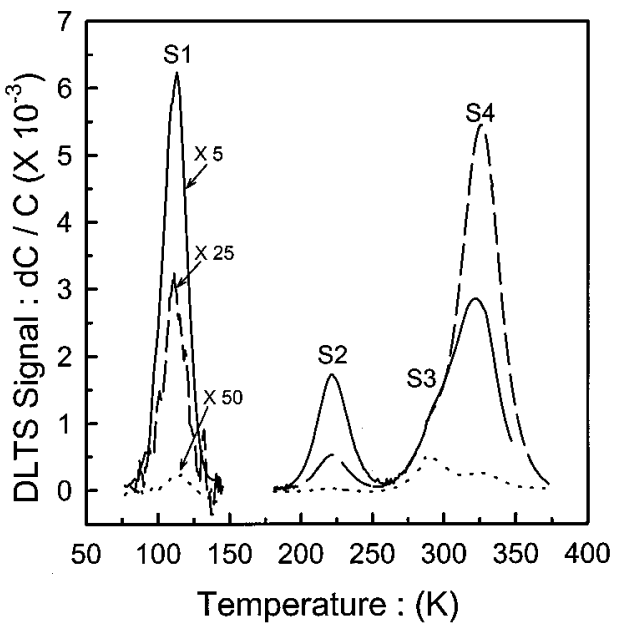

FIG. 3. DLTS spectra obtained from disordered $n$-type GaAs epitaxial layers using undoped (dashed curve), P-doped (solid curve), and Ga-doped (dotted curve) spin-on glass capping layers. The samples were annealed at $900{ }^{\circ} \mathrm{C}$ under Ar flow following baking of the silica layers at $400{ }^{\circ} \mathrm{C}$ for $15 \mathrm{~min}$. The region between 0.35 and $0.57 \mu \mathrm{m}$ was probed in all samples by adjusting the quiescent and filling biases.

when the $n$-GaAs epilayer is annealed without any dielectric capping. ${ }^{2}$ This is in good qualitative agreement with the results shown in Figs. 1(c) and 2(c).

Of more interest are the differences between the defect rates of introduction by undoped and P-doped SOG layers. The concentration of $S 4$ is higher in the $n$-GaAs layer disordered using undoped SOG compared to P-doped SOG, whereas both $S 1$ and $S 2$ show the reverse effect. We have recently provided evidence that $S 4$ is a member of the EL2 family of defects. ${ }^{2,3}$ During RTA the GaAs layer is under compressive stress, ${ }^{5}$ and the formation of $\mathrm{As}_{i}$ and, consequently, $\mathrm{As}_{\mathrm{Ga}}\left(V_{\mathrm{Ga}}+\mathrm{As}_{i} \rightarrow \mathrm{As}_{\mathrm{Ga}}\right)$ is favored. As for $S 1$ and $S 2$, we have proposed that they are related to complexes that involve $\mathrm{As}_{i}$ and $V_{\mathrm{Ga}}$. In particular, we have tentatively identified $S 2$ as the $V_{\mathrm{Ga}}-\mathrm{Si}_{\mathrm{Ga}}$ complex. ${ }^{2,3}$ In light of the results shown in Figs. 2 and 3, we propose that although more $V_{\mathrm{Ga}}$ may be initially produced by the larger outdiffusion of $\mathrm{Ga}$ atoms into the undoped compared to the P-doped SOG layer, a larger fraction of these $V_{\mathrm{Ga}}$ is efficiently converted into the EL2-type defect $S 4$ in the former situation. On the other hand, the lower outdiffusion of Ga atoms into the P-doped layer can be explained by a higher density of the P-doped layer coupled with a stress relaxation effect of this layer during annealing. ${ }^{8}$ This reduced compressive stress also makes the formation of $\mathrm{As}_{\mathrm{Ga}}$, and hence $S 4$, less energetically favorable. Consequently, relatively more $V_{\mathrm{Ga}}$ is retained as shown by the larger concentration of $S 2$ obtained from disordering using the P-doped layer compared to the undoped SOG (Fig. 3).

In light of the results in Figs. 2 and 3 and the above discussion, we are now able to explain the results of impurity-free interdiffusion of GaAs/AlGaAs QWs depicted in Fig. 1. The Ga-doped SOG layer effectively suppresses interdiffusion because the creation of $V_{\mathrm{Ga}}$ is efficiently suppressed. On the other hand, the largest outdiffusion of $\mathrm{Ga}$ atoms is observed in the undoped SOG layer, and yet the extent of interdiffusion is either similar to (QW1) or lower than (QW2) that for the P-doped SOG layer. Although the undoped layer may produce a larger concentration of $V_{\mathrm{Ga}}$ than the P-doped SOG layer, the higher compressive stress imposed on the GaAs layer by the undoped SOG layer during RTA also results in the efficient conversion of $V_{\mathrm{Ga}}$ into $\mathrm{As}_{\mathrm{Ga}}$-type defects. Essentially, the higher concentration of $V_{\mathrm{Ga}}$ which is retained when using P-doped SOG layer produces a larger $\mathrm{Ga}-\mathrm{Al}$ interdiffusion. Our results suggest that the compound effect of the creation of $V_{\mathrm{Ga}}$ and the conversion into $\mathrm{As}_{\mathrm{Ga}}$ under compressive stress is depth dependent, as demonstrated by the different extent of blueshifts in QW1 and QW2 [Figs. 1(a) and 1(b)].

In summary, we have used photoluminescence spectroscopy, x-ray electron spectroscopy, and deep level transient spectroscopy to study the impurity-free disordering process in GaAs-based structures using undoped and doped spin-on silica layers. We have shown that the extent of impurity-free disordering of GaAs-based structures does not depend only on the creation of $V_{\mathrm{Ga}}$ but, maybe more importantly, on their conversion into $\mathrm{As}_{\mathrm{Ga}}$-related defects. Our results have highlighted that a better understanding of the defect reactions related to impurity-free disordering of III-V semiconductors may indeed yield the operative mechanisms behind the interdiffusion process.

Two of the authors (P.N.K.D. and H.H.T.) gratefully acknowledge financial support by the Australian Research Council.

\footnotetext{
${ }^{1}$ For a comprehensive review of impurity-free vacancy interdiffusion, see, for example, Semiconductor Quantum Wells Intermixing, Optoelectronic Properties of Semiconductors and Superlattices, Vol. 8, edited by E. H. Li (Gordon and Breach, Amsterdam, 2000).

${ }^{2}$ P. N. K. Deenapanray, H. H. Tan, C. Jagadish, and F. D. Auret, Appl. Phys. Lett. 77, 696 (2000)

${ }^{3}$ P. N. K. Deenapanray, H. H. Tan, C. Jagadish, and F. D. Auret, J. Appl. Lett. 88, 5255 (2000).

${ }^{4}$ P. N. K. Deenapanray, A. Martin, and C. Jagadish, Appl. Phys. Lett. 79, $2561(2001)$.

${ }^{5}$ A. Pépin, C. Vieu, M. Schneider, H. Launois, and Y. Nissim, J. Vac. Sci. Technol. B 15, 142 (1997).

${ }^{6}$ P. N. K. Deenapanray and C. Jagadish, Electrochem. Solid-State Lett. 4, G11 (2001).

${ }^{7}$ P. N. K. Deenapanray and C. Jagadish, J. Vac. Sci. Technol. B 19, 1962 (2001).

${ }^{8}$ P. Cusumano, B. S. Ooi, A. Saher Helmy, S. G. Ayling, A. C. Bryce, J. H. Marsh, B. Voegele, and M. J. Rose, J. Appl. Phys. 81, 2445 (1997).

${ }^{9}$ G. Li, S. J. Chua, S. J. Xu, X. C. Wang, A. Saher Helmy, M.-L. Ke, and J. H. Marsh, Appl. Phys. Lett. 73, 3393 (1998).

${ }^{10}$ L. Fu, P. N. K. Deenapanray, H. H. Tan, C. Jagadish, L. V. Dao, and M. Gal, Appl. Phys. Lett. 76, 837 (2000).

${ }^{11}$ L. Fu, R. W. v. d. Heijden, H. H. Tan, C. Jagadish, L. V. Dao, and M. Gal, Appl. Phys. Lett. 80, 1171 (2002).

${ }^{12}$ E. V. K. Rao, A. Hamoudi, Ph. Krauz, M. Juhel, and H. Thibierge, Appl. Phys. Lett. 66, 472 (1995).
} 